\title{
Correction to: Schlafen-11 expression is associated with immune signatures and basal-like phenotype in breast cancer
}

\author{
Edoardo Isnaldi ${ }^{1}$. Domenico Ferraioli ${ }^{1,2}$. Lorenzo Ferrando ${ }^{1}$. Sylvain Brohée ${ }^{3}$. Fabio Ferrando ${ }^{1,4} \cdot$ Piero Fregatti $^{1,4}$. \\ Davide Bedognetti ${ }^{5}$. Alberto Ballestrero ${ }^{1,4}$. Gabriele Zoppoli ${ }^{1,4}$ (i)
}

Published online: 12 July 2019

(c) Springer Science+Business Media, LLC, part of Springer Nature 2019

\section{Correction to: Breast Cancer Research and Treatment https://doi.org/10.1007/s10549-019-05313-w}

In the original publication of the article, the funding information was incorrectly published. The corrected funding statement is given in this correction article.

Funding This work (DB work: gene expression analysis) was supported in part by Qatar National Research Fund (NPRP10-0126-170262 "Identification of molecular determinants of breast cancer immune responsiveness by integrative genome-scale analysis", awarded to DB).

The original article can be found online at https://doi.org/10.1007/ s10549-019-05313-w.

Gabriele Zoppoli

gabriele.zoppoli@unige.it

1 Department of Internal Medicine (DiMI), University of Genoa and Ospedale Policlinico San Martino, Viale Benedetto XV, 6, 16132 Genoa, Italy

2 Comprehensive Cancer Center Leon Berard, Lyon, France

3 Institut de Pathologie Et de Génétique a.s.b.l, Charleroi, Belgium

4 Ospedale Policlinico San Martino IRCCS per l'Oncologia, Genoa, Italy

5 Sidra Medical Center, Doha, Qatar
Publisher's Note Springer Nature remains neutral with regard to jurisdictional claims in published maps and institutional affiliations. 